\title{
Occupational medicine type 2 diabetes screening in Luxembourg
}

\author{
Macovei M ${ }^{1 *}$, Radermecker RP4, Majery $N^{1}$, Schritz $A^{2}$ and Rusu $D^{3,4}$ \\ ${ }^{1}$ Service de Santé au Travail Multisectoriel, Luxembourg \\ ${ }^{2}$ Luxembourg Institute of Health, Luxembourg \\ ${ }^{3}$ SPMT ARISTA External Service for Prevention and Protection at Work, Belgium \\ ${ }^{4}$ University of Liège, Belgium
}

\begin{abstract}
Background: Type 2 diabetes, accounting for $90 \%$ of diabetes cases, is often diagnosed several years after its onset, once the complications are already present, resulting in considerable economic loss. Early and effective detection should avoid or delay the consequences it may have.

Aims: This study aims to optimize diabetes screening in the Grand Duchy of Luxembourg, among the active population on the labor market.

Methods: A cross-sectional descriptive study was conducted using a participatory survey, which included workers between the ages of 18 and 65 , who have never been diagnosed with diabetes. Employees responded to the FINDRISC questionnaire and had a measurement of capillary blood glucose and an glycated hemoglobin (HbA1c) assay. The aim was to establish the level of predictability and reliability of the FINDRISC questionnaire and the correlation with capillary blood glucose and $\mathrm{HbA1c}$.

Results: The univariable model of the FINDRISC score (threshold $>=10$ ) seems to be the most suitable for the detection of dysglycemia in an occupational medicine setting.

Conclusions: Employees with a FINDRISC score $<10$ will be re-evaluated at the next periodical examination, those with a FINDRISC score $>=10$ and a low-risk job position will be referred for follow-up to their family physicians; in addition, for those with FINDRISC score > = 10 and a high-risk job position an HbA1c assay will be performed at the occupational medicine clinic.
\end{abstract}

\section{Introduction}

Diabetes constitutes a major problem in public health with type 2 representing $90 \%$ of all cases of diabetes. Because of symptoms less marked than in type 1 diabetes, it is often diagnosed several years after its onset, once the complications are already present resulting in considerable economic loss. Early and efficient detection should avoid or delay the incidence of these side effects and thereby reduce the economic burden of direct health spending and indirect costs related to absenteeism and disability pensions [1].

Screening for type 2 diabetes mellitus (DM2) should be performed in adults of all ages who are overweight or obese and have one or more risk factors for diabetes [2]: physical inactivity, family history (a first degree relative), ethnical background with DM2 high risk (LatinAmerican, Asian or African), having delivered a child with a birth weight of more than $4 \mathrm{~kg}$ (91b), HDL cholesterol $<35 \mathrm{mg} / \mathrm{dl} \pm$ triglycerides $>250 \mathrm{mg} / \mathrm{dl}$, high blood pressure ( $\geq 140 / 90 \mathrm{~mm} \mathrm{Hg}$ or under treatment), glycated hemoglobin (HbA1c) $\geq 5,7 \%$, glucose intolerance or impaired fasting glucose, medical conditions associated with insulin resistance: severe obesity, acanthosis nigricans, medical history of cardio-vascular diseases. Screening should begin at age 45 [2]. If the test is normal, it should be repeated at least every 3 years.

The screening methods for dysglycemia patients are: risk assessment using the FINDRISC score [3], use of the recommended screening tests [2]: fasting blood glucose $\geq 100 \mathrm{mg} / \mathrm{dL}$ (5.6 mmol/L), HbAlc $\geq 5.7$ (39 $\mathrm{mmol} / \mathrm{mol}$ ), random blood glucose $\geq 200 \mathrm{mg} / \mathrm{dL}$ (11.1 mmol/L) for patients with symptoms of hyperglycemia.

The method of screening for diabetes currently used in occupational medicine in the Grand Duchy of Luxembourg is the detection of glycosuria by using a urine dipstick. With this screening method, because of the very high threshold (blood glucose $>180 \mathrm{mg} / \mathrm{dl}$ ) from which glycosuria is detected, the "pre-diabetic" and some of the diabetic patients remain undetected.

A meta-analysis of 178 published articles also found that urine dipsticks had insufficient sensitivity to be used as a screening tool in an asymptomatic population. [4]. In addition, false-positive or falsenegative results decrease even more the utility of this method as a screening tool for dysglycemia.

The medical examinations in occupational medicine should be performed during the working hours, a reason why a fasting glucose

*Correspondence to: M. Macovei, Service de Santé au Travail Multisectoriel, 32 rue Glesener, L-1630 Luxembourg, Tel: +352 400942 1226; E-mail: mihai.macovei@stm.lu

Key words: type 2 diabetes, pre-diabetes, screening, prevention, occupational medicine

Received: July 29, 2019; Accepted: August 19, 2019; Published: August 26, 2019 
test or an oral glucose tolerance test (OGTT) remain inapplicable in the occupational medicine settings.

In this context, the glycated hemoglobin seems to be the best choice among all the available tests [5]. The FINDRISC score was developed in Finland following the need for a non-invasive tool for screening for type 2 diabetes [6]. It is the most commonly used questionnaire for detecting undiagnosed diabetes [7].

Therefore, in our study, we have tried to identify the best test or combination of tests that could lead to a better screening of diabetes in an occupational health setting.

\section{Methodology}

This is a cross-sectional descriptive study conducted at STM (a multisector occupational medicine service in Luxembourg) by participatory survey (Findrisc questionnaire), measurement of capillary blood glucose (CBG) and blood sample for the determination of the glycated hemoglobin (HbA1c).

The FINDRISC questionnaire focuses on the risk factors of developing a type 2 diabetes (age, family history, waist circumference, physical activity, diet, treated hypertension, history of carbohydrate intolerance, body mass index, etc).

The minimum sample size of 323 participants was calculated using the following hypothesis: alpha risk at 0.05 , a percentage of prediabetic and diabetic adults in the Grand Duchy of Luxembourg of approximately $30 \%$ [8], a precision of 5\% [9]. Taking into account a history of average response rate of $60 \%$, a minimum of 539 patients needed to be asked to participate in the study. In the end, 1120 individuals were asked to participate in the study and 506 of them accepted.

Population description: adults (18-65 years old) who have never been diagnosed with diabetes were included in the study. A written informed consent was requested from all the participants. Adults who have already been diagnosed with diabetes mellitus (type 1 or type 2 ) have been excluded.

The study took place over 3 months, between April and June 2017. All patients / employees who met the inclusion criteria, were informed and asked to agree to complete the FINDRISC questionnaire and to perform a capillary blood glucose measurement as well as to have withdrawn a blood sample for an HbAlc assay. The data from each questionnaire, as well as the biological results, were recorded into our clinic medical database.

The data of those who participated in the survey and the additional information on those who refused to participate were provided, in pseudonymized form, to the Luxembourg Institute of Health (LIH). For individuals participating in the study, median, minimum and maximum values were calculated for continuous variables. For categorized or binary variables, the number of individuals and the percentage for each category were calculated.

Descriptive statistics were calculated for those who did not agree to participate in the study compared to those who participated. The same statistics were calculated for the dysglycemic and non-dysglycemic participants according to HbAlc.

Differences between groups were tested with the non-parametric Wilcoxon-Mann-Witney test for continuous variables if there were only two groups and the Chi-Square test for categorized and binary variables. Values of $\mathrm{P}<0.05$ were considered significant. No alpha correction was used to for multiple testing.
Univariable logistic regression was performed to investigate associations between gender, FINDRISC score, CBG, and glycosuria with dysglycemia classification. In addition, receiver operating characteristic (ROC) functions were calculated and the area under the curve (AUC) was calculated. A multivariable logistic regression was performed, after selecting the variables for the model with the best fit, to predict dysglycemia based on individual characteristics and dysglycemia tests.

The FINDRISC score was not included in models where characteristics such as age, BMI and abdominal perimeter were used, because these variables are already included in this score. As a result, other models were created using the FINDRISC score and the covariables that were not used to calculate the score. The Youden index was calculated for the logistic regression with binary variables for dysglycemia classified by HbAlc, with CBG and FINDRISC score as predictor variables. The maximum of the Youden index gives the best combination of sensitivity and maximum specificity to find a cut-off value of the FINDRISC score.

In addition, weighted Cohen's Kappa index was calculated to measure the inter-rater agreement between the classification of dysglycemia using HbA1c versus capillary blood glucose (CBG). As already mentioned in the previous section, variable selection for a multivariable logistic regression model was performed to find a model best suited to the data.

The classification of dysglycemia in relation to normoglycemia was defined as outcome variable. Age, gender, BMI, abdominal perimeter, capillary glycaemia, and glycosuria were used as predictors. No significant difference between the two groups (participants versus nonparticipants) regarding age, BMI or high-risk job positions was found. In contrast, more men refused to participate compared to women $(59 \%$ vs. $52 \%$, p-value: 0.03 ).

We have then calculated the participation rate according to different characteristics: age, category of BMI and high-risk job position. We characterized the total sample and non-dysglycemic versus dysglycemic participants by descriptive statistics.

The selection of the variables (type "forward, backward" and "combined forward and backward") was performed. The models with the lowest AIC value ("Akaike Information Criterion") [10] were chosen as models with the best fit.

All tests and calculations were performed using the statistical software R (Version 3.2.2). This study was presented and validated by the National Committee of Ethics and Research (CNER) in the Grand Duchy of Luxembourg in December 2016.

\section{Results}

The population of non-participants was compared to that of the participants by analyzing the descriptive statistics of the characteristics measured in the two groups. The categorized variables (age group, gender, body mass index category, and having a high-risk job position) are presented in Table 1.

The participants were represented by $48 \%$ women and $52 \%$ men. The median age of all participants was 36 (range: $18-64$ years). The median BMI was $25.2 \mathrm{~kg} / \mathrm{m} 2$ (range: $16.8-52.8 \mathrm{~kg} / \mathrm{m} 2$ ). The median FINDRISC score was 6 (range: 0-21).

The median age of normoglycemic participants was 34 years (with a minimum of 18 years and a maximum of 62 years) compared to the 
Table 1. Descriptive statistics of categorical variables grouped by participation

\begin{tabular}{|c|c|c|c|}
\hline & Non-Participants & Participants & \\
\hline Age & N (\%) & N (\%) & p-value \\
\hline$<35$ & $238(47 \%)$ & $285(46.4 \%)$ & \\
\hline $35-44$ & $131(25.9 \%)$ & $166(27 \%)$ & \\
\hline $45-54$ & $100(19.8 \%)$ & $123(20 \%)$ & \\
\hline $55-64$ & $36(7.1 \%)$ & $40(6.5 \%)$ & \\
\hline$>64$ & $1(0.2 \%)$ & $0(0 \%)$ & \\
\hline Gender & & & \\
\hline Women & $209(41.3 \%)$ & $294(47.9 \%)$ & \\
\hline Men & $297(58.7 \%)$ & $320(52.1 \%)$ & \\
\hline BMI & & & \\
\hline Normal & $226(45.1 \%)$ & $268(43.8 \%)$ & \\
\hline Overweight & $188(37.5 \%)$ & $249(40.7 \%)$ & \\
\hline Obese & $87(17.4 \%)$ & $95(15.5 \%)$ & \\
\hline High-risk job* & & & \\
\hline Yes & $311(61.5 \%)$ & $385(62.7 \%)$ & \\
\hline No & $195(38.5 \%)$ & $229(37.3 \%)$ & \\
\hline
\end{tabular}

*a high-risk job represents any job position involving an activity that may seriously endanger the safety and health of the worker himself or of the other workers or third parties.

median age of 46.5 years (with a minimum of 24 years and a maximum of age 64) for dysglycemic participants. Participants classified as dysglycemic were significantly older ( $\mathrm{p}$-value: $<0.001$ ) compared to participants classified as normoglycemic.

In the normoglycemic group, the distribution of women and men was the same (50\%: 50\%), whereas in the dysglycemic group, there were slightly more men $(61 \%)$ than women. Nevertheless, no significant difference between the two groups was found (p-value: 0.16 ).

In the dysglycemic group, a significantly higher BMI was observed compared to the normoglycemic group (30.4 versus $24.6, \mathrm{p}$ value $<0.001)$. The participants classified as dysglycemic presented a FINDRISC score significantly higher compared to the normoglycemic group (12 against 6 , p-value: $<0.001$ ).

The Youden index of 0.462 maximum has defined the threshold value for the FINDRISC score when predicting HbAlc-defined dysglycemia (Figure 1) The sensitivity and specificity for detecting dysglycemia was $68.5 \%$ and $77.7 \%$ respectively for a threshold value $>=$ 10 (Figures 2 and 3).

\section{Discussion}

Our study confirms a series of results published in the scientific literature. The detection of glycosuria by urine dipstick shows poor discrimination (AUC 0.566) as a screening test for dysglycemia (Figure $3)$.

The use of random capillary blood glucose as a screening test for dysglycemia seems to be inappropriate, given the low discrimination power with an AUC of 0.676 (Figure 2) and the inter-rater agreement between $\mathrm{HbAlc}$ and capillary blood.

Regarding of having relatives (parents, children or siblings) with diabetes, $17.6 \%$ in the normoglycemic group gave an affirmative response compared to $41.2 \%$ in the dysglycemic group ( $\mathrm{p}$-value: $<0.001$ ). Asked if they were diagnosed with glucose intolerance in the past, $17 \%$ in the dysglycemic group answered "yes", compared with $4 \%$ in the normoglycemic group ( $\mathrm{p}$-value: $<0.001$ ).

The agreement of classification of dysglycemia defined by HbAlc and capillary blood glucose (CBG) were compared using weighted
Cohen's Kappa index. A weighted index of 0.24 (95\% CI: [0.1; 0.37]) could be interpreted as very weak agreement. For each univariable model, their estimated area under the curve (AUC), can be found in Figures 1, 2 and 3 below. Glucose, with weighted Cohen's Kappa index of 0.24 (very weak agreement).

The analysis of models taking into account several variables (gender, FINDRISC score, capillary blood glucose) shows almost the same (good) discrimination capacity as the FINDRISC score alone. Given the unreliability of capillary blood glucose to identify dysglycemic individuals, as well as the absence of gender differences (men / women), the FINDRISC score model alone would appear to be the most suitable for the initial screening of dysglycemia among an active population at work.

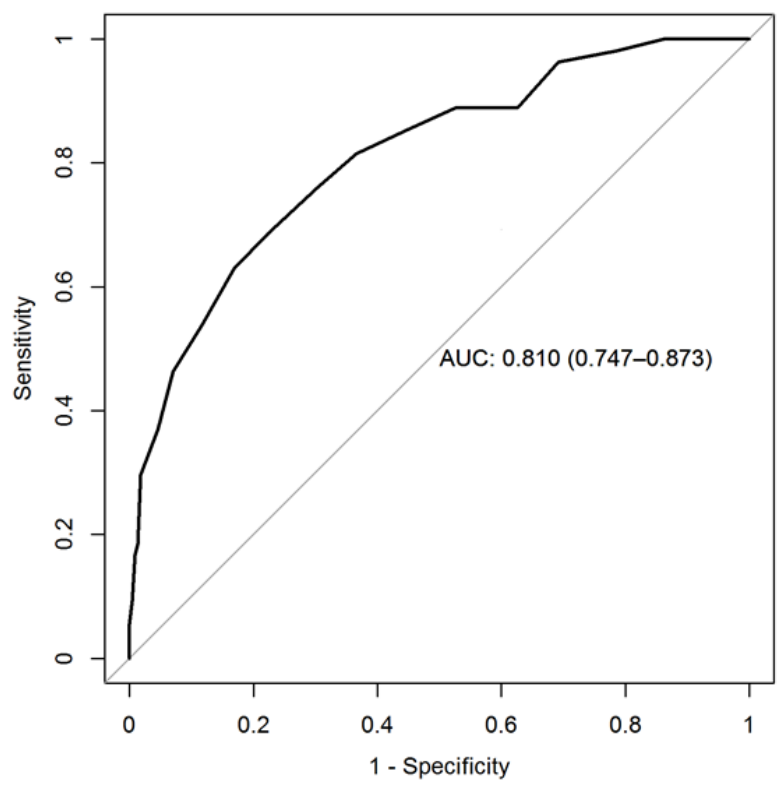

Figure 1. ROC: Dysglycemia / FINDRISC

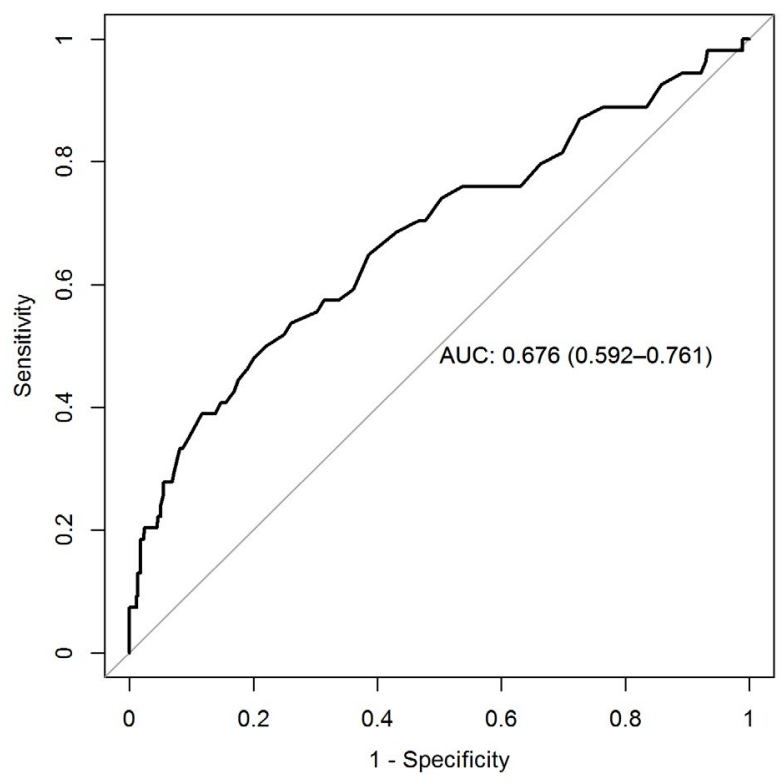

Figure 2. ROC: Dysglycemia / CBG 




Figure 3. ROC: Dysglycemia / Glycosuria

The FINDRISC score of 10 for dysglycemia screening is consistent with data from other studies [11,7]. The results confirm that random capillary blood glucose [12] and the urinary dipstick glycosuria [13] are not suitable as screening tests for dysglycemia.

The active population at work (18-65 years) often escapes regular control for the preventive purpose in primary care, the occupational medicine physician often remaining the only health professional in contact with this population.

The possibility of screening for this population could eliminate certain biases (advanced age, co-morbidities, etc.) present in the context of screenings performed in other circumstances: general practitioners' offices, hospitals, etc.

Also, the ease of screening for pre-diabetes / diabetes, using the FINDRISC questionnaire (and biological means such as the HbAlc assay), as well as the orientation of patients at risk towards their general practitioner for management and monitoring, may reduce the consequences of late diagnosis of this disease with insidious onset.

The number of participants classified, as dysglycemic (54) was small compared to the total number of participants (healthy worker effect?). This unbalanced design could lead to a lower power than the detection of possible associations between variables and predictions.

The results obtained should be verified in other studies.

The classification of dysglycemia defined by HbAlc would appear to have a lower sensitivity than other methods of detecting dysglycemia, such as fasting blood glucose or the oral glucose tolerance test $[14,15]$, but given the particularity of medical examinations in occupational medicine (where the employees are examined during the working hours), HbAlc remains the only approved method $[16,17]$ for such a screening.

\section{Conclusion}

The univariable model of the FINDRISC score (threshold $>=$ 10) seems to be the most suitable for the detection of dysglycemia in occupational medicine. The additional variables (gender, capillary blood glucose etc.) only bring a modest benefit.
The algorithm of screening for dysglycemia in an active population at work, aged between 18 and 65, could be the following (Figure 4):

- Employees with a FINDRISC score $<10$ will be re-evaluated at the next periodical medical examination,

- Those with a FINDRISC score $\geq 10$ and a job position defined as lowrisk will be referred to their family physician for care and regular follow-up,

- Employees with a FINDRISC score $\geq 10$ and a high-risk job position will have performed a blood test for HbAlc assay (a faster reception of the result is necessary for the fitness to work decision) and, afterwards, they will be referred to their general practitioner for further care and regular monitoring.

All employees who will be examined in occupational medicine at our clinic will receive detailed information on the risks and prevention of type 2 diabetes.

Given the progressive aging of the population in Europe and the increase in the retirement age, as well as the decrease in the age of onset of type 2 diabetes, more effective screening for dysglycemia in occupational medicine (as well as in other medical fields), will only bring benefits for both employees and their employers, as well as for national health systems.

\section{Key points}

- The active population at work (18-65 years old) often escapes regular control for the preventive purpose in primary care, the occupational medicine physician often remaining the only health professional in contact with this population.

- The FINDRISC score (threshold $>=10$ ) seems the most suitable for the detection of dysglycemia in an occupational medicine setting.

- Given the progressive aging of the population in Europe and the increase of the age of retirement, as well as the decrease in the age of onset of type 2 diabetes, more effective screening for dysglycemia in occupational medicine will only bring benefits for both employees and their employers, as well as for national health systems.

\section{Funding}

This work was supported by STM (Service de Santé au Travail Multisectoriel, Luxembourg).

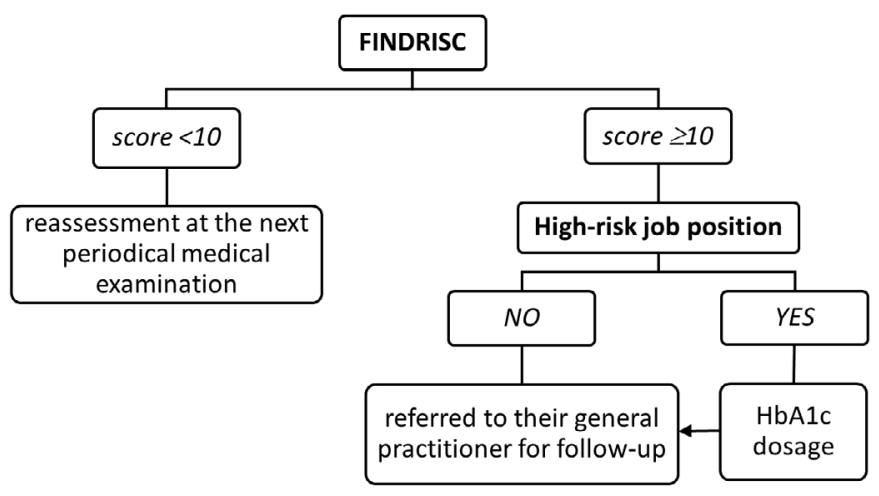

Figure 4. Proposed algorithm for dysglycemia screening: general population at work 


\section{Conflicts of interest}

The authors declare that they have no conflicts of interest.

\section{References}

1. Organisation mondiale de la Santé (2016) Rapport mondial sur le diabète, Genève.

2. American Diabetes Association (2016) Classification and Diagnosis of Diabetes. Diabetes Care 39: 13-22.

3. Luxembourg Institute of Health (2008) Le diabète au Luxembourg, www.statistiques. public.lu.

4. Association Luxembourgeoise du Diabète (2014) Le guide du diabète, www.ald.lu

5. American Diabetes Association (2016) Standards of medical care in diabetes. Diabetes Care 39: 1-106.

6. Walker HK, Hall WD, Hurst JW (1990) Clinical Methods: The History, Physical, and Laboratory Examinations, 3rd edition, Butterworths.

7. Wei OY, Teece S (2006) Best evidence topic report. Urine dipsticks in screening for diabetes mellitus. Emerg Med J 23: 138. [Crossref]

8. Sepulchre E, Lutteri L, Cavalier E, Guerci B, Radermecker RP, (2012) A propos de l'hémoglobine glyquée : Les limites de son interpretation. Revue Médicale de Liège 133-138.
9. Lindström J, Tuomilehto $\mathrm{J}$ (2003) The diabetes risk score: a practical tool to predict type 2 diabetes risk. Diabetes Care 26: 725-731. [Crossref]

10. Bilous R, Donnelly R (2010) Handbook of Diabetes, Wiley-Blackwell.

11. Skerjanc A (2001) Sickness absence in diabetic employees. Occup Environ Med 58: 432-436. [Crossref]

12. Akaike H (1974) A new look at the statistical model identification. IEEE Transactions on Automatic Control 716-723.

13. Landis JR, Koch GG (1977) The measurement of observer agreement for categorical data. Biometrics 33: 159-174. [Crossref]

14. Zhang L, Zhang Z, Zhang Y, Hu G, Chen L (2014) Evaluation of finnish diabetes risk score in screening undiagnosed diabetes and prediabetes among U.S. adults by gender and race: NHANES 1999-2010. PLoS One 9: e97865. [Crossref]

15. Tirimacco R, Tideman PA, Dunbar J, Simpson PA, Philpot B, et al. (2010) Should capillary blood glucose measurements be used in population surveys? International Journal of Diabetes Mellitus 24-27.

16. American Academy of Family Physicians (2010) Diabetes Mellitus: Screening and Diagnosis, Am Fam Physician 81: 863-870.

17. Organisation mondiale de la Santé (2003) Screening for Type 2 Diabetes, Geneva.

Copyright: $₫ 2019$ Macovei M. This is an open-access article distributed under the terms of the Creative Commons Attribution License, which permits unrestricted use, distribution, and reproduction in any medium, provided the original author and source are credited. 